Meta

Journal des traducteurs

Translators' Journal

\title{
Continuidad de los textos: Metaficción en un cuento de Cortázar y en su traducción sueca
}

\section{Cecilia Alvstad et Åse Johnsen}

Volume 57, numéro 3, septembre 2012

URI : https://id.erudit.org/iderudit/1017082ar

DOI : https://doi.org/10.7202/1017082ar

Aller au sommaire du numéro

Éditeur(s)

Les Presses de l’Université de Montréal

ISSN

0026-0452 (imprimé)

1492-1421 (numérique)

Découvrir la revue

Citer cet article

Alvstad, C. \& Johnsen, å. (2012). Continuidad de los textos: Metaficción en un cuento de Cortázar y en su traducción sueca. Meta, 57(3), 592-604.

https://doi.org/10.7202/1017082ar
Résumé de l'article

Le présent article contient une analyse de la traduction suédoise de la nouvelle Continuidad de los parques écrite par l'auteur argentin Julio Cortázar et traduite en suédois par Jan Sjögren. Cette nouvelle est un excellent exemple de la métafiction, car elle joue sur la relation entre un lecteur imaginé et le lecteur réel. À l'aide d'une illusion esthétique, Cortázar crée un suspense laissant ses lecteurs aux prises avec des questions sans réponse tout au long de la lecture. L'analyse montre que le suspense est affaibli et fonctionne différemment dans la version suédoise de l'histoire. En changeant le titre, ainsi que d'autres parties mineures, mais néanmoins significatives, du texte, le traducteur transfère sa propre interprétation du texte dans la traduction suédoise. La structure narrative est altérée et, par conséquent, le lecteur de la version suédoise ne peut accéder aux multiples interprétations auxquelles le texte, dans la version originale espagnole de Cortázar, donne accès. 


\title{
Continuidad de los textos: Metaficción en un cuento de Cortázar y en su traducción sueca
}

\author{
CECILIA ALVSTAD \\ Universidad de Oslo, Oslo, Noruega \\ cecilia.alvstad@ilos.uio.no \\ ÅSE JOHNSEN \\ Universidad de Bergen, Bergen, Noruega \\ ase.johnsen@if.uib.no
}

\section{RÉSUMÉ}

Le présent article contient une analyse de la traduction suédoise de la nouvelle Continuidad de los parques écrite par l'auteur argentin Julio Cortázar et traduite en suédois par Jan Sjögren. Cette nouvelle est un excellent exemple de la métafiction, car elle joue sur la relation entre un lecteur imaginé et le lecteur réel. À l'aide d'une illusion esthétique, Cortázar crée un suspense laissant ses lecteurs aux prises avec des questions sans réponse tout au long de la lecture. L'analyse montre que le suspense est affaibli et fonctionne différemment dans la version suédoise de l'histoire. En changeant le titre, ainsi que d'autres parties mineures, mais néanmoins significatives, du texte, le traducteur transfère sa propre interprétation du texte dans la traduction suédoise. La structure narrative est altérée et, par conséquent, le lecteur de la version suédoise ne peut accéder aux multiples interprétations auxquelles le texte, dans la version originale espagnole de Cortázar, donne accès.

\begin{abstract}
This article analyzes the Swedish translation of the short story Continuidad de los parques written by the Argentine author Julio Cortázar and translated into Swedish by the translator Jan Sjögren. This short story is an excellent piece of metafiction as it plays with the relation between a fictional reader and the real reader. By creating an aesthetic illusion Cortázar leaves the reader in a tense state with a number of unanswered questions during the reading of the text. The analysis shows that this state of tension is weakened and that it works differently in the Swedish version of the story. By changing the title and other minor, but substantial, parts of the text, the translator transfers his own interpretation of the text into the Swedish translation. The narrative structure is altered and, as a result of this, the reader of the Swedish version does not have access to the same multiple interpretations that Cortázar's Spanish text opens up for.
\end{abstract}

\section{MOTS-CLÉS/KEYWORDS}

traduction littéraire, métafiction, titre

literary translation, metafiction, title

\section{Introducción - objetivos}

Los títulos de obras literarias y de películas con frecuencia están sujetos a cambios al ser traducidos. Estos cambios pueden tener motivaciones varias, desde aspectos relacionados con el márketing hasta las diferentes connotaciones que despierta un título en una cultura determinada. En muchos casos el cambio también puede ser una elección del traductor motivada por preferencias personales y/o estéticas. 
Algunos ejemplos de cambios en la traducción de títulos de novelas latinoamericanas al sueco, los encontramos en los siguientes casos: la novela Los pasos perdidos de Alejo Carpentier ${ }^{1}$, que al sueco se ha traducido por Den förlorade porten [el portón perdido] ${ }^{2}$, o la novela del mismo autor El siglo de las luces ${ }^{3}$, traducida como Detta upplysta tidevarv [este siglo iluminado] ${ }^{4}$. También es el caso del cuento objeto del presente análisis, Continuidad de los parques ${ }^{5}$, de Julio Cortázar, que en sueco se titula Drama $i$ park [drama en parque] ${ }^{6}$. Los tres textos mencionados fueron traducidos por el traductor sueco Jan Sjögren durante la década de los 60. En el primer título de Carpentier se cambia los pasos por el portón, perdiéndose de esa manera una importante referencia a Les pas perdus de André Breton 7 . En el segundo se transforma la referencia temporal a «el siglo de las luces», que en el título original se refiere a la Ilustración, mientras que el determinante demostrativo este del título sueco aparentemente sitúa el eje temporal en nuestro tiempo. En lo que se refiere al cuento de Cortázar se ha cambiado continuidad por drama y además el plural definido de parques se ha convertido en singular indefinido, park [parque].

Los títulos, como bien ha señalado Genette (1987: 7), no sólo presentan sino que también condicionan la lectura y, por tanto, traen consecuencias para la lectura del texto en su totalidad. En este artículo llevaremos a cabo una lectura comparativa de la versión original y la traducción sueca de Continuidad de los parques, tomando como punto de partida los cambios en el título. El objetivo es indagar en las posibles razones por las que se ha transformado el título en la traducción al sueco y relacionar el cambio con la traducción en su totalidad. Analizaremos las diferencias entre traducción y original para mostrar cómo la traducción de algunos elementos verbales influye en la lectura, y, por tanto, también en las posibles interpretaciones del texto. De esa manera discutiremos tanto las causas como los efectos de los cambios introducidos.

Una traducción siempre es el resultado de decisiones tomadas durante el proceso de traducción (Levy 1967; Toury 1995). Estas decisiones se ven condicionadas por múltiples factores. Entre ellos el texto fuente, que de un modo u otro ejerce poder sobre lo que puede formular el traductor, y la relación entre las lenguas y culturas involucradas, que hasta cierto punto determina cómo el traductor puede formularlo. Lo último incluye también lo que es lingüísticamente posible formular en la lengua meta así como lo que obligatoriamente no ha de formularse dada la construcción de la misma (Jakobson 1959/1989).

Entre los otros aspectos que pueden ejercer influencia sobre cómo se formula un texto traducido encontramos los marcos de referencia del traductor, los cuales influyen tanto en su interpretación como en sus preferencias estéticas, ambos elementos de importancia en el momento en que se formula el texto traducido. También puede entrar en juego lo que el traductor considera que son las expectativas del público lector, ya que un traductor puede introducir cambios en una traducción con el fin de que sea mejor recibida por el público lector que imagina, es decir que el traductor busca seguir las normas de expectación (ver «expectancy norm» en Chesterman 1997).

También debe notarse que los condicionantes del texto, lengua y cultura fuente, así como las posibilidades y limitaciones ofrecidas por la lengua y cultura meta, no son absolutos: están involucrados varios condicionantes pero entre ellos también se crea un espacio amplio para la creatividad del traductor (Malmkjær 2005: x). 
Como la traducción es una actividad que necesariamente involucra decisiones condicionadas por tantos factores diferentes y además está condicionada por la creatividad del traductor, siempre habrá diferencias entre una traducción y su texto fuente, así como entre varias traducciones del mismo texto (Vidal Claramonte 2005: 9-27). Las traducciones son interesantes porque no son copias de un original (Hermans 1999: 95) y es precisamente por esta razón por lo que indagaremos en los resultados de las decisiones tomadas en cuanto a algunas palabras, con el fin de analizar cómo influye en las posibles interpretaciones del texto en su totalidad.

Las decisiones que se toman sobre una traducción son generalmente realizadas por una serie de profesionales tales como el editor, el traductor y el corrector. Como bien ha señalado Toury (1995: 183), no es posible distinguir en un análisis textual, como el que pretendemos llevar a cabo en el presente artículo, quién de los agentes involucrados es el responsable de una decisión tomada. El traductor es por ello en muchos estudios un «hypothetical construct, i.e. a functional entity mediating between two existing texts, rather than a definite person» (Toury 1995: 183). Al referirnos al traductor en el presente artículo nos referimos por ello al conjunto de personas que pueden haber estado involucradas en las decisiones tomadas.

Según hemos señalado arriba, mostraremos en este artículo cómo la traducción de algunas palabras traen consecuencias para la lectura de otras partes del texto y, por tanto, también para las posibles interpretaciones. Para poder hacer esto nos serviremos de una distinción hecha por Nord entre unidades de traducción horizontales y verticales. Nord (1998: 66) indica que se suele entender por unidad de traducción «la unidad de la lengua o del texto de partida tratada por el traductor en el proceso de traducción». Señala que los estudiosos no se han puesto de acuerdo sobre el tamaño de tal unidad ni del nivel lingüístico en el que se ubica, esto es, «si debería constituirse por morfemas, palabras, colocaciones o frases, oraciones o incluso textos enteros» (Nord 1998: 66). Nord discute en detalle una serie de definiciones de la unidad de traducción hechas por otros traductólogos y señala que al definir dicha unidad, todos ellos tienen en común que se mueven en un plano horizontal del texto. Nord plantea una contrapropuesta: que en vez de definir la unidad de traducción como un elemento lineal (de alcance mayor o menor) se defina la unidad de traducción como elementos funcionales que puedan aparecer verticalmente en diferentes lugares en un mismo texto y menciona a modo de ejemplo las funciones referencial, expresiva, apelativa y fática (Nord 1998: 71).

Si bien estas funciones no son aplicables sin ajustes a un texto literario, queremos aquí retomar la idea de Nord y probar la hipótesis de que hay un tema central en el cuento Continuidad de los parques que funciona verticalmente en el texto, y que por tanto se construye por elementos aislados a lo largo de todo el texto, desde el título hasta las palabras finales y que, por ello, conviene realizar el análisis desde un punto de vista vertical y no lineal.

Este tema central es el de la metaficción, el más tratado en los numerosos análisis de este cuento (e.g., Genette 1972; Lewis 1978; Di Gerónimo 2006; Palmer 2009). Volveremos sobre las lecturas de otros estudiosos en el apartado 3, pero ya aquí podemos adelantar que el protagonista del cuento está tan profundamente sumergido en la lectura de un libro que empieza a formar parte de él. Constatamos por ello que hay un elemento metanarrativo muy marcado en el cuento. 
La traducción sueca de Continuidad de los parques fue publicada por primera vez en 1969 en el volumen Slut på leken [Final del juego], junto con otros cuentos de Cortázar. Este libro fue lo primero que se publicó en sueco de Julio Cortázar. El cuento se volvió a publicar por la casa editorial Modernista en $2007^{8}$, en una edición de dos tomos que reúnen los cuentos de Cortázar que ya habían sido traducidos al sueco. A propósito de esto, la revista Karavan también publicó el cuento a principio de 20089. En la versión publicada en sueco en 2007 se hicieron algunos cambios en comparación con la versión de 1969, por ejemplo los extranjerismos finca y mayordomo se sustituyeron por palabras suecas como lantgård y förvaltare, pero en general se trata de la misma traducción. Basamos el presente análisis en la versión publicada en 1969, pero todas las observaciones que hacemos a lo largo del análisis son válidas para las versiones retocadas de esta traducción.

\section{Sinopsis del cuento}

En Continuidad de los parques hay dos historias que aquí, por cuestiones de comodidad llamaremos A y B. En la historia A un hombre está leyendo una novela, sentado en un sillón de terciopelo verde en un cuarto que da hacia un parque de robles. En la historia B seguimos los acontecimientos de esa novela: dos amantes se encuentran en una cabaña del monte, repasando los últimos detalles de una conspiración asesina. Al separarse, ella se va hacia el norte y él hacia el sur, con un puñal en la mano. Siguiendo las instrucciones de la mujer, el amante llega a la casa, entra en la sala donde ve al hombre sentado en un sillón de terciopelo verde leyendo una novela.

Aparentemente la historia A funciona como marco y la historia B como historia enmarcada. Sin embargo, cuando en el último párrafo (que se narra desde la perspectiva de la historia B), el amante entra en la sala y ve la espalda del protagonista de la historia A, quien simultáneamente lee precisamente esta historia, se rompe el esquema de historia marco e historia enmarcada. El final es a la vez un encuentro simbiótico entre las dos historias (A y B) y un retorno desde la historia B a la historia A. Por tanto, nos encontramos ante un elemento lúdico típicamente cortazariano.

\section{La interpenetración de lo real y lo irreal}

El aspecto lúdico es un tema muy central en la obra cortazariana y en muchos de sus cuentos se crea un juego entre la realidad y la irrealidad. Según Luis Eyzaguirre (1986: 182-183), los cuentos de Cortázar se pueden agrupar en tres grupos según el tipo de relación que existe entre los planos de la realidad y de la irrealidad. En el primer grupo el plano de la irrealidad invade el de la realidad y destruye la rutina diaria. Como ejemplo de este grupo Eyzaguirre menciona La casa tomada y Carta a una señorita en París, entre otros. En el segundo grupo sucede una inversión de los planos de manera que se crea una inseguridad sobre qué es lo real. Los cuentos Axolotl y La noche boca arriba son ejemplos de este grupo. En el tercer grupo encontramos el cuento objeto del presente análisis, y sobre este grupo señala Eyzaguirre:

En la tercera categoría se inscriben los cuentos que muestran la interpenetración de los planos de lo real y lo irreal y un traspaso de las identidades individuales. Son relatos que ilustran la fusión de lo real y lo extraño en una realidad otra. En el espacio imaginario del texto se posibilita esta fusión y el correspondiente traspaso de identidad con una bien definida creación de la figura del doble. (Eyzaguirre 1986: 182) 
Y es exactamente esa figura del doble la que crea la metaficción de Continuidad de los parques. Según Eyzaguirre: «[e]l lector que lee de espaldas a la puerta y el amante que, en el texto de la novela, mata a su rival, y en el espacio y tiempo de la lectura mata al absorto lector con quien el personaje literario se ha fusionado» (Eyzaguirre 1986: 182). En otras palabras, tal como hemos señalado arriba, la realidad (la historia A) y la irrealidad (la historia B) se fusionan al final del cuento.

El juego entre la historia A y B en Continuidad de los parques se consigue mediante medidas estilísticas. Julia E. Palmer (2009) ha demostrado cómo las dos historias del cuento se definen por el uso de los tiempos verbales y más adelante nosotras hacemos énfasis en cómo la repetición de ciertas palabras también contribuye a la fusión de las dos historias.

En lo que a los tiempos verbales se refiere, Palmer señala que el pretérito indefinido domina en la historia A mientras que el pretérito imperfecto lo hace en la historia B. A medida que las dos historias se acercan para luego fusionarse, el uso del pretérito indefinido va introduciéndose en la historia B y el cuento culmina en un párrafo que carece de verbos conjugados. Mediante este uso no muy tradicional de la lengua, Cortázar crea, según Palmer, más tensión: «The effect of deleting all verb forms from the final part of the narration increases the tension. The image is much like a film in which the physical progression of the killer through the house is told in a series of jump cuts» (Palmer 2009: 210). Palmer argumenta que la falta de verbos conjugados en la última parte se puede interpretar de manera que las historias se han fusionado:

The disappearance of all verb forms at the end of story two essentially creates a third story or narration, one on which the distance between story one and story two has disappeared because both are now part of the same story. (Palmer 2009: 210)

Palmer añade además una cuarta historia, la que el lector del cuento crea al llegar al final: es el lector mismo quien tiene que descubrir la fusión entre las dos historias.

Según Palmer, el uso de los tiempos verbales es un recurso estilístico que sirve para diferenciar y luego unir las dos historias. La posibilidad que tiene el español de emplear estos dos tiempos verbales está ausente en muchas otras lenguas, como por ejemplo el sueco. De esta manera, el lector sueco no puede guiarse por los tiempos verbales ni para diferenciar las dos historias ni para fusionarlas. No obstante, la fusión de las dos historias se lleva a cabo también por otros recursos textuales que sí son fáciles de verter también a la lengua sueca. A lo largo de la narración hay una serie de elementos léxicos que unen las dos historias. No solamente reaparece el lector de la historia A al final de la historia B, o en la fusión de ambas, sino que también hay una serie de vocablos que se encuentran tanto en la historia A como en la B. Estos elementos crean un paralelismo entre las dos historias y ayudan al lector a interpretar el final como una fusión de las dos historias. Las modificaciones introducidas pueden parecer pequeñas a primera vista. Desde un punto de vista horizontal (según la terminología de Nord 1998: 71) de hecho no son cambios grandes, pero sí son importantes desde un punto de vista vertical, ya que son modificaciones que conciernen a los elementos de enlace por los cuales las dos historias se fusionan en una. Cuando en la versión sueca estos elementos léxicos no son traducidos de manera consecuente, se priva por ello al lector sueco de la posibilidad de crear la cuarta historia. 


\section{Los títulos}

El título del cuento, Continuidad de los parques, señala que hay dos o más parques, así como una relación entre ellos. Es un título sugestivo que invita al lector a adivinar cuáles son los parques y el tipo de relación que existe entre ellos. El título sueco Drama i park [drama en parque], en comparación, es un título concreto, indica que el lector va a ser testigo de un drama que tiene lugar en un parque.

La palabra parque(s) es una palabra clave en ambos títulos. También aparece en los dos textos. A pesar del plural del título (los parques), en el original aparece sólo una mención explícita a un parque. La mención figura en relación con la descripción de la casa donde se encuentra el protagonista de la historia A: miraba hacia el parque de los robles. En otras palabras, la única aparición de la palabra parque en el original forma parte de la historia marco (A). Si hay otro parque, es el lector quien lo ha de identificar.

En cambio, en la versión sueca hay dos menciones explícitas de parque en el texto. Una forma parte de la historia A y corresponde a la arriba citada. La otra forma parte de la historia B en la cual un mundo de hojas secas y senderos furtivos se traduce como parkens torra löv och dolda stigar, esto es, mundo se traduce como parque. De esta manera la versión sueca, que solamente menciona un parque en el título, introduce dos en el cuerpo del cuento.

La palabra continuidad señala la existencia de un proceso. Posiblemente remita a la relación entre las dos historias contadas en el cuento, la historia A y la historia B. En este caso, identificamos la palabra parques del título con las dos historias que se cuentan. Sin embargo, el tema se complica ya que sólo se menciona un parque en el cuerpo del texto del original. Para que el lector pueda relacionar la «continuidad de los parques» con la continuidad de las dos historias, es necesario, por tanto, que el lector o bien haya identificado un parque también en la historia $\mathrm{B}$ o bien que lo interprete como una pista textual más metafórica. Al traducir mundo por park [par$q u e]$, el traductor sueco opta claramente por la primera alternativa, y de esa manera, imposibilita a los lectores de su texto pasar por el proceso de duda por el que pasan los lectores del texto original.

Tal como hemos señalado en la introducción, las elecciones de un traductor siempre se ven condicionadas por las diferencias contrastivas entre las lenguas involucradas. Por lo tanto, es relevante preguntarse si, visto desde una perspectiva lingüística, hubiese sido posible traducir el título Continuidad de los parques de otra manera. Para averiguar esas posibilidades o limitaciones lingüísticas hemos estudiado también las traducciones a otras lenguas escandinavas y hemos visto que los demás traductores escandinavos han optado por traducir el título de una manera mucho más parecida al original:

(1) Parkar som går over i kvarandre (noruego nynorsk) [Parques que se sobreponen]

(Cortázar 1969/2002, traducido por Fløgstad; retrotraducido por las autoras $)^{10}$

(2) Sammenhengen mellom parkene (noruego bokmål) [La relación/La continuidad entre los parques]

(Cortázar 1969/1994, traducido por Risvik en 1970; retrotraducido por las autoras) ${ }^{11}$ 
(3) Parkernes kontinuitet (danés)

[La continuidad de los parques]

(Cortázar 1960/1976, traducido por Hasselbalch; retrotraducido por las autoras) $)^{12}$

Como vemos, los tres títulos traducidos reproducen tanto la idea de continuidad, como la idea de parques en plural. Podemos, por tanto, concluir que hubiese sido posible reproducir ambas ideas también en sueco, ya que las lenguas escandinavas se parecen mucho tanto en la sintaxis como en el léxico. El haber optado por Drama $i$ park es una decisión no impuesta por las limitaciones de la lengua meta.

\section{Las estructuras}

En el texto de Cortázar sólo en las últimas líneas del cuento se unen las dos historias. Antes de estas últimas líneas, la historia B pertenece claramente a un mundo ficticio dentro de la ficción. Esto se señala mediante frecuentes alusiones al acto de lectura en la historia A: la ilusión novelesca lo ganó casi en seguida, Gozaba del placer casi perverso de irse desgajando línea a línea y Palabra a palabra, absorbido por la sórdida disyuntiva de los héroes. Son alusiones metaficticias que llaman la atención tanto sobre el acto de lectura que ocurre en el texto como sobre el acto de lectura de los mismos lectores del cuento de Cortázar. Además, estas alusiones contribuyen a crear una diferencia cualitativa entre la historia A y la historia B. La historia A incentiva al lector a reflexionar sobre la lectura y por ello se puede percibir como más central que la historia $B$.

Si por un momento dejamos de lado el final del cuento (en el cual, según hemos señalado arriba, se rompe el esquema de historia marco-historia enmarcada), proponemos la siguiente representación gráfica de la versión de Cortázar (Cuadro 1):

CUADRO 1

Relación entre historia A e historia B en la versión original

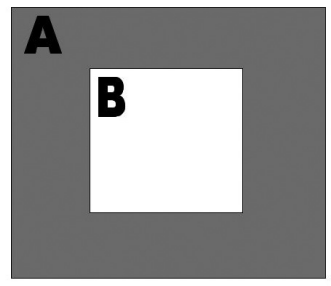

El Cuadro 1 muestra cómo, en la versión original, la historia A enmarca la historia B. Tal como hemos señalado más arriba, la versión sueca, opta por sustituir la palabra continuidad por drama, además de traducir el plural de parques por el singular de park, cambios que contribuyen a transformar la relación entre las historias A y B. Ya no hay una relación entre varios parques, sino que hay un drama que se desarrolla en un parque, y el drama forma parte de la historia B. De esa manera el foco central pasa desde la historia A hasta la historia B. Este modo de lectura se fortalece además por la omisión de varias de las alusiones metaficticias de la versión original: la ilusión novelesca se traduce como romanens intrig [la trama de la novela] y palabra a palabra se elimina. 
Podemos representar gráficamente la relación entre las dos historias en la traducción sueca de la manera señalada en el Cuadro 2 (y tampoco en este cuadro tenemos en cuenta el final del cuento):

CUADRO 2

Relación entre historia A e historia B en la traducción

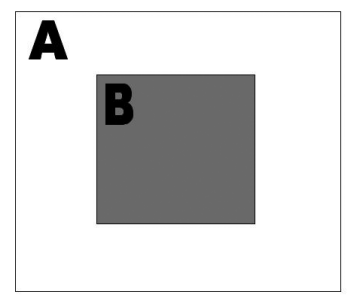

El Cuadro 2 ilustra que, antes de llegar al final del cuento, se pone de relieve la historia B frente a la historia A. El contraste gris/blanco en ambas figuras hay que entenderlo de forma relativa. Lo que queremos resaltar es que la historia B destaca más en la versión sueca que en la versión original.

\section{Los finales}

Hacia el final del cuento nos encontramos en el nivel narrativo de la historia $\mathrm{B}$, el amante entra, puñal en mano, en una casa. Antes de llegar a la última oración del cuento (de su versión original), la historia A sigue enmarcando a la B. En la última oración se altera esta relación entre las dos historias. Según hemos señalado en la sinopsis, se produce simultáneamente un retorno a la historia marco (A) y una simbiosis entre las dos historias A y B.

Técnicamente esta alteración se produce por la repetición de una serie de palabras ya empleadas antes en el texto: puerta, ventanales, alto respaldo, sillón, terciopelo verde. Cuando se emplean por primera vez, estas palabras forman parte de la historia A. Al final del cuento pertenecen a la historia B. La oración final en su totalidad se lee como sigue: La puerta del salón, y entonces el puñal en la mano, la luz de los ventanales, el alto respaldo de un sillón de terciopelo verde la cabeza del hombre en el sillón leyendo una novela (nuestro subrayado, nuestra negrita).

Nuestro subrayado resalta las palabras repetidas que con anterioridad se han usado en la historia A. La palabra puñal antes se ha empleado en la historia B. Se produce por ello en esta oración una unión entre las dos historias. Una historia parece yuxtaponerse a la otra y se produce cierta inseguridad sobre el estado de la cuestión: ¿Es esta puerta la misma puerta que se ha mencionado anteriormente en la historia A? ¿Son los mismos ventanales? Cuando además se menciona un sillón de alto respaldo tapizado con terciopelo verde ya no parece haber duda. La última pista, que parece ser la decisiva, no se encuentra en el empleo repetido de estas palabras sino cuando se introduce el hombre que lee la novela. Al emplearse la forma definida el hombre se señala que este hombre ya es conocido por el lector, quien, por consiguiente, es invitado a identificarlo con el protagonista de la historia A. De manera gráfica proponemos la siguiente representación del desenlace de este cuento (Cuadro 3): 
CUADRO 3

El desenlace de la versión original

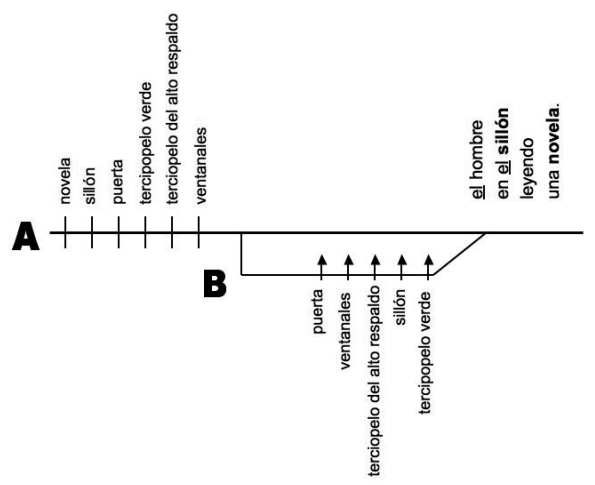

Se produce un paralelismo entre las dos historias por el empleo repetido de dichas palabras. Con la mención de el hombre el paralelismo se convierte en unión. Después de esta oración final, ya no es válida la relación entre A y B según fue representada en el Cuadro 1. El marco narrativo parece consumirse, o bien, posiblemente, empieza a formar parte de la historia enmarcada. Una propuesta de representación gráfica es la siguiente:

CUADRO 4

La relación entre la historia A y B en el cuento en su totalidad (versión original)

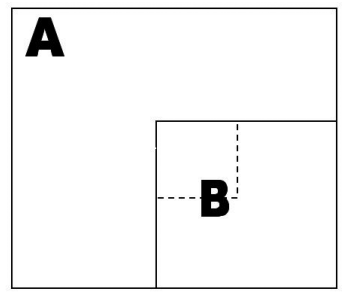

En este cuadro se ilustra cómo la historia marco se fusiona con la historia enmarcada. Ninguna de las dos historias desaparece sino que la historia A sigue existiendo junto a la historia B. Parece que la historia B invade el marco, o, posiblemente, se sobrepone a la historia A. La figura puede interpretarse de ambos modos.

Como demostraremos a continuación, estas representaciones gráficas del final (Cuadros 3 y 4) no son adecuadas para el final de la traducción sueca. Curiosamente, en la traducción sueca se produce un desenlace distinto. Éste se debe a varios factores que no sólo se encuentran en la última oración del cuento sino a lo largo de todo el texto, el título incluido. Ya hemos mencionado uno de ellos: la sustitución de continuidad por drama en el título. Otro factor decisivo es que el traductor sueco ha optado por soluciones que contribuyen a que las dos historias se acerquen la una a la otra más arriba en el texto. La más obvia de estas decisiones es la sustitución de mundo por park [parque]. Con esta sustitución se establece un paralelismo léxico entre la historia A y B, las dos historias se acercan, y de esa manera el traductor sueco puede anticipar el desenlace del cuento. 
También hay otro punto en la traducción sueca en el que se anticipa el final. El protagonista de la historia A lee su novela y se deja absorber por la ficción: Palabra a palabra, absorbido por la sórdida disyuntiva de los héroes, dejándose ir hacia las imágenes que se concertaban y adquirían color y movimiento. El traductor sueco traduce este pasaje de la siguiente manera:

(4) Fascinerad av den smutsiga uppgörelsen mellan huvudpersonerna lät han sig ryckas med så intensivt att gestalterna fick färg och blev levande för honom [Fascinado por el sucio ajuste de cuentas entre los protagonistas se dejó llevar tan intensamente que los personajes adquirían color y cobraban vida para él]

(Cortázar 1960/1969, traducido por Sjögren; retrotraducido por las autoras)

Los efectos de la traducción de este pasaje son complejos. Por una parte se elimina un elemento metaficticio (palabra a palabra). Por otra parte, se introduce otro, y este nuevo elemento metaficticio es mucho más evidente: los personajes cobran vida para él. De esa manera, se anticipa el encuentro entre los protagonistas de las historias A y B, encuentro que en la versión original se produce en el desenlace del cuento. Tal como hemos visto, uno de los personajes de B, de hecho cobra vida para el lector de la novela en los últimos renglones del cuento.

Estas dos diferencias afectan a la relación entre las historias A y B. La eliminación de palabra a palabra, junto con otras eliminaciones parecidas, hace que las diferencias estilísticas entre las dos historias se debiliten. Esto es debido a que las referencias metaficticias pertenecen únicamente a la historia A. En otras palabras, las dos historias se acercan la una a la otra. Con la formulación blev levande för honom [cobraban vida para él] se produce un acercamiento todavía más evidente entre las dos historias, parecido al efecto de la traducción de mundo por park [parque]. Proponemos la siguiente representación gráfica:

\section{CUADRO 5}

En la traducción sueca la historia B se acerca antes en el texto a la historia A.

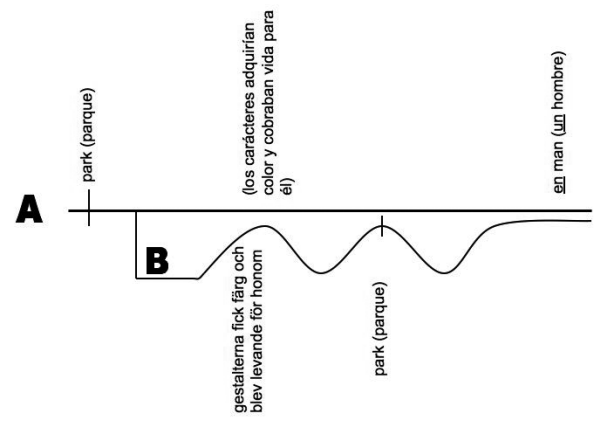

En la traducción sueca la historia B se acerca antes en el texto a la historia A (al emplear las expresiones suecas blev levande för honom [cobraban vida para él] y park [parque]). En el final de la traducción la fusión entre las dos historias no se produce igual de nítidamente. De esa manera se anticipa el paralelismo entre las dos historias A y B en la traducción sueca. Sin embargo, a diferencia de lo que sucede en la versión original, en la traducción este paralelismo no desemboca en una unión. Las dos historias continúan siendo paralelas, lo cual se desprende del Cuadro 5. 
Tal como hemos señalado, la oración final es decisiva para la interpretación del cuento y es en ella en donde se unen las dos historias. La traducción al sueco de esa oración es la siguiente:

(5) Där efter dörren till arbetsrummet (och då med kniven i handen). Ljuset från de stora fönstren - det höga ryggstödet på en länstol klädd med grön sammet - och huvudet på en man som satt och läste en roman...

[Después la puerta del estudio (y entonces con el puñal en la mano). La luz de los ventanales $-\mathrm{y}$ el alto respaldo de un sillón tapizado de terciopelo verde - y la cabeza de un hombre sentado leyendo una novela...]

(Cortázar 1960/1969, traducido por Sjögren; retrotraducido por las autoras)

Como podemos ver, la oración se divide en dos oraciones y el uso de signos es diferente: en la traducción se emplean guiones y la oración se termina con puntos suspensivos, mientras que en el original no.

Tal como hemos dicho, en el original se emplea la repetición en la historia B de palabras que anteriormente se han usado en la historia A. En la traducción, este recurso sólo se reproduce de forma parcial. Sillón se traduce como favoritfåtölj cuando aparece en la historia A pero por länstol cuando aparece en la historia B y por eso se debilita la posibilidad de identificar los sillones como uno solo. La identificación se hace todavía más difícil ya que la última aparición de la palabra sillón se elimina completamente en la traducción.

La mencionada unión tampoco se da en la traducción, puesto que el hombre se ha traducido como en man [un hombre]. El artículo indefinido indica que este personaje es desconocido para el lector.

En otras palabras, en la traducción sueca hay también un paralelismo entre las dos historias. Este paralelismo se hace evidente incluso antes en la traducción que en el original. Sin embargo, al contrario del original, en el cual el paralelismo se convierte en una unión de las dos historias, nos quedamos en la traducción con una mera alusión a una unión posible entre las dos historias. Esta unión posible se produce por la repetición de las palabras puerta, ventanales, terciopelo verde y alto respaldo que sí se repiten en ambas historias.

En la traducción ni se produce la simbiosis final de la versión original, ni se produce una vuelta al marco narrativo. Además, el cuento sueco no se cierra con la oración final, sino que hay un desenlace abierto. Los puntos suspensivos en la traducción indican que hay algo más en ese final, pero es el lector quien tiene que encontrarlo. Podríamos incluso decir que la historia B se escapa del marco narrativo, lo cual intentamos ilustrar en el Cuadro 6:

\section{CUADRO 6}

En la traducción, la historia marco pierde el control sobre la historia enmarcada que se escapa del marco narrativo de la historia $\mathrm{A}$.

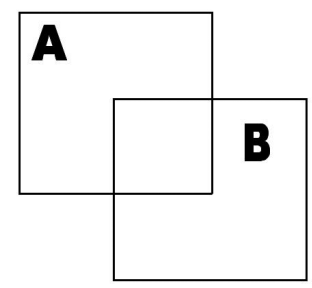


El que la traducción termine en puntos suspensivos subraya además lo dramático en vez de lo metanarrativo. En la traducción de los parques del título tenemos un cambio del plural al singular, en el punto final un cambio contrario, del singular al plural. Sin embargo, estos dos cambios de número, que inician y cierran la traducción, crean efectos similares. Hacen que el cuento esté más focalizado sobre la trama y se disminuya el énfasis sobre las dimensiones metanarrativas del cuento.

Las representaciones gráficas de la estructura del cuento propuestas arriba para la versión original, funcionan también para las otras tres traducciones escandinavas. A pesar de que los otros traductores a su vez hacen cambios con respecto al original, estos cambios no afectan tan claramente a la estructura del cuento y a las posibles interpretaciones. Este hecho tiene que ver con la traducción del título así como con la traducción de los otros elementos que contribuyen a lo metaficticio del cuento, entre ellas, el final.

La manera en que se han traducido al sueco estos ejemplos afecta al cuento en su totalidad. El cambio del título se ve reforzado por otros cambios introducidos por el traductor sueco a lo largo del texto. Los cambios no son necesariamente decisiones conscientes por parte del traductor; no obstante, producen en su conjunto una modificación importante de lo metanarrativo del cuento. El jugar con los límites entre ficción y realidad y entre lectura y vida, tan típicamente cortazariano, no se actualiza de la misma manera en la traducción sueca.

\section{Conclusiones}

Tal como hemos visto en lo arriba expuesto, el cambio del título es uno de los varios elementos verticales que contribuyen al carácter metaficticio del cuento. El traducir Continuidad de los parques por Drama i park contribuye a que se cambien las posibilidades de interpretación del cuento y es un cambio que junto a las otras modificaciones de lo metafictivo afecta a la totalidad del texto. Entre otras transformaciones, cambia la estructura narrativa del cuento, más precisamente la relación entre historia marco e historia enmarcada. Además se transforma la temática del cuento ya que el juego lúdico entre realidad y ficción se debilita. También la traducción sueca tiene un tema metaficticio, pero tiene menos importancia, y la manera de presentarlo es además más tradicional y menos trasgresora que la que hay en la versión de Cortázar. Dado que no existen limitaciones lingüísticas que puedan justificar los cambios, parece tratarse de elecciones que o bien tienen que ver con la interpretación del texto por parte del traductor o bien de lo que el traductor cree que pueden ser las expectativas de sus lectores. Es un cuento que se puede leer y disfrutar en sueco, pero se diferencia mucho del original. De hecho se diferencia tanto del original que para un lector que sólo conozca la traducción sueca no serían comprensibles los análisis literarios presentados en el apartado tres.

En cuanto al porqué de los cambios, podemos constatar que puesto que sólo hemos analizado un cuento aislado no podemos saber si los cambios son típicos del traductor o también de su entorno, esto es, de la tradición traductora de la cultura meta en ese momento. Para averiguarlo habría que analizar los elementos metaficticios también en otros cuentos de Cortázar y en la obra de otros autores que trabajan con elementos y temáticas de metaficción. 


\section{NOTAS}

1. Carpentier, Alejo (1959): Los pasos perdidos. México: Compañía General de Ediciones.

2. Carpentier, Alejo (1959/1963): Den förlorade porten. (Traducido por Jan SJögren) Stockholm: Bonnier.

3. Carpentier, Alejo (1963): El siglo de las luces. La Habana: Ediciones Revolución.

4. Carpentier, Alejo (1963/1965): Detta upplysta tidevarv. (Traducido por Jan SjöGren) Stockholm: Bonnier.

5. Cortázar, Julio (1960/1974): Continuidad de los parques. In: Julio Cortázar, Final del juego. $16^{e}$ ed. Buenos Aires: Editorial Sudamericana, 9-11. La primera edición de Final del juego fue publicado en 1956. Según Yurkievich (2003: 1119), Continuidad de los parques fue publicado por primera vez en la revista Grillo de Papel (núm. 4, 1960). En 1964 fue incluido en la edición Sudamericana de Final del juego.

6. Cortázar, Julio (1960/1969): Drama i park. (Traducido por Jan SJöGren) In: Julio Cortázar, Slut på leken: noveller. Stockholm: Bonnier, 51-52.

7. BREton, André (1924): Les pas perdus. Paris: Gallimard.

8. Cortázar, Julio (1960/2007): Drama i park. (Traducido por Jan SJögren) In: Julio Cortázar, Samlade noveller 1. Stockholm: Modernista, 367-368.

9. CORTÁZAR, Julio (1960/2008): Drama i park. (Traducido por Jan SJöGren) Karavan. 1:40-41.

10. CoRTÁzAR, Julio (1960/2002): Parkar som går over i kvarandre. (Traducido por Kjartan FløgSTAD) In: Den må du lese! 24 favorittfortellinger. Oslo: Gyldendal, 101-102.

11. CoRTÁZar, Julio (1960/1970): Sammenhengen mellom parkene. (Traducido por Kjell RisviK) In: Julio Cortázar, Seremonier. Oslo: Gyldendal, 7-8.

12. Cortázar, Julio (1960/1976): Parkernes kontinuitet. (Traducido por Iben Hasselbalch) Gyldendals magasin. 21:109-110.

\section{REFERENCIAS BIBLIOGRÁFICAS}

Chesterman, Andrew (1997): Memes of Translation: The Spread of Ideas in Translation Theory. Amsterdam/Philadelphia: John Benjamins.

Di Gerónimo, Miriam (2006): Laberintos verbales de autoficción y metaficción en Borges y Cortázar. Cuadernos del CILHA. 7/8:56-89.

Eyzaguirre, Luis (1986): Modos de lo fantástico en cuentos de Julio Cortázar. En: Coloquio Internacional Lo lúdico y lo fantástico en la obra de Cortázar. Centre de recherches LatinoAmericaines Université de Poitiers. Madrid: Editorial Fundamentos, 177-184.

Genette, Gérard (1972): Figure III. Paris: Éditions du Seuil.

Genette, Gérard (1987): Seuils. Paris: Éditions du Seuil.

Hermans, Theo (1999): Translation in Systems. Descriptive and System-oriented Approaches Explained. Manchester: St. Jerome.

Jakobson, Roman (1959/1989): On Linguistic Aspects of Translation. In: Andrew Chesterman, ed. Readings in Translation Theory. Helsinki: Oy Finn Lectura, 53-60.

Levy, Jiři (1967): Translation as a Decision Process. In: s.n., To Honor Roman Jakobson: Essays on the Occasion of his Seventieth Birthday. The Hague: Mouton, 1171-1182.

Lewis, Davis K. (1978): Truth in Fiction. American Philosophical Quarterly. 15:37-46.

MALMKJÆR, Kirsten (2005): Linguistics and the Language of Translation. Edinburgh: Edinburgh University Press.

NorD, Christiane (1998): La unidad de traducción en el enfoque funcionalista. Quaderns. Revista de traducció. 1:65-77.

Palmer, Julia E. (2009): Verbs, Voyeurism and the Stalker Narrative in Cortázar's 'Continuidad de los parques'. Romance Quarterly. 56(3):207-216.

Toury, Gideon (1995): Descriptive Translation Studies and Beyond. Amsterdam/Philadelphia: John Benjamins.

Vidal Claramonte, África (2005): En los límites de la traducción. Granada: Editorial Comares. Yurkievich, Saúl (2003): Antecedentes de esta edición. In: Julio CortázAr, Obras completas. Barcelona: Opera Mundi, 1117-1120. 